\title{
Incidental Collision Tumor of Hepatocellular Carcinoma and Neuroendocrine Carcinoma
}

\author{
Düriye Betül Yılmaz, Zeynep Bayramoğlu, Gülşah Ünay, Erdem Ayık, \\ Cumhur İbrahim Başsorgun and Gülsüm Özlem Elpek*
}

Department of Pathology, Akdeniz University Medical School, Konyaalti/Antalya, Turkey

\begin{abstract}
The composite tumors of the liver are very rare, including the coexistence of HCC (hepatocellular carcinoma) with NEC (neuroendocrine carcinoma). The rare occurrence of these tumors necessitates more reported cases in order to fully understand their clinical characteristics, behaviors and treatments. Herein is described an incidental collision tumor of HCC-NEC, along with a review of the literature focusing on their clinicopathological findings and prognosis. The tumor presented here was found incidentally in the hepatectomy specimen of a 56-yearold man who had undergone liver transplantation for rapidly progressive liver failure because of alcoholic hepatitis and cirrhosis. Imaging and laboratory examinations did not demonstrate tumor-related findings. During macroscopic examination, two sharply defined and distinctive areas $(1.7 \mathrm{~cm}$ and $0.6 \mathrm{~cm}$ dimension respectively) were detected among the cirrhotic nodules. The characteristic histopathological features and immunohistochemical findings allowed a diagnosis of HCCNEC to be made. There was no evidence of recurrence and metastasis after 10 months following surgery. The present case and review revealed that these tumors are frequently found in older ages and males. Although serum markers are valuable in the discrimination of malignant tumors, their absence cannot completely rule out composite HCC-NEC. Diagnosis requires a comprehensive histopathological evaluation together with immunohistochemistry. The NEC component might influence the treatment strategy and eventually the outcome of the patient. In conclusion, the rare occurrence of HCC-NEC and the lack of diagnostic clinical signs and symptoms do not exclude their consideration in the differential diagnosis of liver tumors, especially in patients with the chronic liver disease.
\end{abstract}

Citation of this article: Yılmaz DB, Bayramoğlu Z, Ünay G, Ayık E, Başsorgun CI, Elpek GÖ. Incidental collision tumor of hepatocellular carcinoma and neuroendocrine carcinoma. J Clin Transl Hepatol 2018;6(3):339-344. doi: 10.14218/ JCTH.2017.00076.

Keywords: Liver neoplasm; Hepatocellular carcinoma; Neuroendocrine carcinoma.

Abbreviations: AFP, alpha-fetoprotein; HCC, hepatocellular carcinoma; NEC, neuroendocrine carcinoma; PIVKA-II, protein induced by vitamin $\mathrm{K}$ deficiency or antagonists-II.

Received: 29 November 2017; Revised: 03 April 2018; Accepted: 06 April 2018 *Correspondence to: Gülsüm Özlem Elpek, Department of Pathology, Akdeniz University Medical School Hospital, Dumlupinar Street, Pinarbasi District, Konyaalti/Antalya-07070, Turkey. Tel: +90-242-249-6389, E-mail: elpek@akdeniz. edu.tr

\section{Introduction}

Two different tumors may coexist simultaneously in several organs. These tumors are classified as combined type or collision type according to their histopathological features. ${ }^{1}$ In the liver, both types are rare. While the combined type tumors represent $2.0-3.6 \%$ of all primary hepatic malignancies, ${ }^{2,3}$ collision tumors are even rarer, with an incidence of $0.1-1 \%{ }^{2,4} \mathrm{~A}$ great majority of these tumors consist of HCC (hepatocellular carcinoma) accompanied by a cholangiocarcinoma. ${ }^{2-4}$ The occurrence of HCC with other tumors is scarce, including the coexistence of HCC with neuroendocrine carcinoma (NEC). Indeed, a limited number of such cases have been reported recently, warranting the description of new cases to better understand their behavior. In a study involving 1235 cases of liver cancer, the incidence of these tumors was found to be $0.46 \%{ }^{5}$ Here, we report a case with an incidental collision tumor of HCC with NEC (HCC-NEC) and provide a review of the literature focusing on their clinicopathological findings and prognosis.

\section{Case report}

The patient was a 56-year-old man who was followed up for alcoholic hepatitis and cirrhosis for 4 years. His medical history included obesity, diabetes mellitus and Crohn's disease, which had been continuing for 7 years. He was admitted to our emergency clinic with amnesia, debility, apathy and aphasia. Physical examination revealed a mild abdominal distension related to ascites. Imaging findings of the liver (ultrasonography, doppler, and computed tomography indicated irregular contours and diffuse granular heterogeneity of the parenchyma (Fig. 1). Laboratory tests demonstrated hypoalbuminemia $(0.49 \mathrm{~g} / \mathrm{dL})$, hyperbilirubinemia (total bilirubin: $2.55 \mathrm{mg} / \mathrm{dL}$ and direct bilirubin: $1.80 \mathrm{mg} / \mathrm{dL}$ ) and hyperammonemia (197.61 $\mu \mathrm{g} / \mathrm{dL})$. Tests for viral and autoimmune markers were negative. Aspartate aminotransferase (30 U/L), alanine aminotransferase (45 U/L) and alkaline phosphatase (65 U/L) were in normal ranges. The serum alpha-fetoprotein (AFP) level was $2.8 \mathrm{ng} / \mathrm{mL}$. Other tumor markers, such as carcinoembryonic antigen and carbohydrate antigen 19-9 were also within the normal range.

Because of the rapid progress of the liver failure, a liver transplantation was performed. Serum albumin $(0.60 \mathrm{~g} / \mathrm{dL})$, aspartate aminotransferase $(26 \mathrm{U} / \mathrm{L})$, alanine aminotransferase $(13 \mathrm{U} / \mathrm{L})$ and alkaline phosphatase $(73 \mathrm{U} / \mathrm{L})$ were tested preoperatively. There was no evidence of hepatic failure, recurrence or metastasis in the 10 months following surgery. 


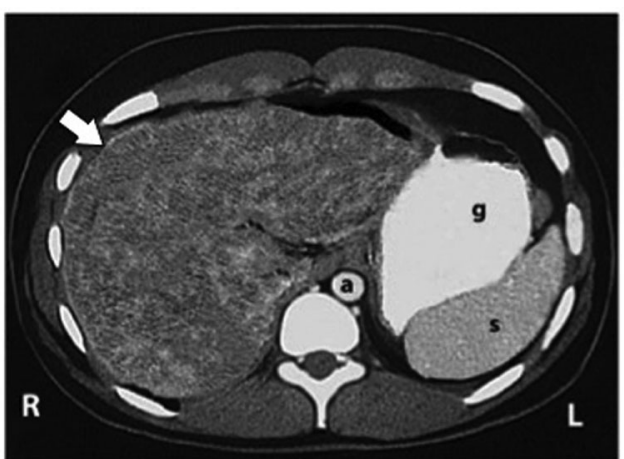

Fig. 1. Abdominal CT of the case revealed irregular contours and diffuse granular heterogeneity of the liver parenchyma, supporting cirrhosis. No mass was observed. Abbreviations: a, aorta; Arrow, liver; L, left; g, stomach; R, right; s, spleen.

Moreover, no serological endocrine markers were detected (i.e. chromogranin A, gastrin, glucagon and vasoactive intestinal polypeptide) after the pathological diagnosis.

\section{Methods}

The liver specimen was fixed in $10 \%$ buffered formalin, embedded in paraffin, cut into $4-\mu m$-thick sections, and stained with hematoxylin and eosin. Immunohistochemistry was performed on deparaffinized tissue sections using the avidin-biotin-peroxidase method and a Dako AutoStainer (Carpinteria, CA, USA). After antigen retrieval, the following panel of primary antibodies was applied: Hep Par1 (hepatocellular antigen, 1:50; Dako), CD34 (1:200; Dako), synaptophysin (1:10; Dako), chromogranin (1:50; Dako), CK7 (1:1,500; Dako), CK19 (1:50; Dako), CD56 (1:50; Vision BioSystem), MIB-1 (1:100; Dako) and $\beta$-Catenin (1:250; Dako).

\section{Gross findings}

The resected specimen consisted of a $18 \times 14 \times 9 \mathrm{~cm}$ hepatectomy with an attached gallbladder, consisting of $800 \mathrm{~g}$. The external surface was coarsely nodular. Cut sections showed well-demarcated nodules, measuring from less than $1 \mathrm{~cm}$ to up $3 \mathrm{~cm}$ in greatest dimensions. Between these nodules, a well circumscribed and demarcated but non-encapsulated region of masses was present. The region was composed of two sharply defined and distinctive areas, including a green area with $1.7 \times 1 \times 1.6 \mathrm{~cm}$ dimension and the second being a distinctly yellow-tan colored area with lesions having the largest dimension of $0.6 \mathrm{~cm}$ (Fig. 2). No satellite lesions were identified. The gallbladder was normal.

\section{Histopathological findings and immunohistochemistry}

The green and nodular component consisted of tumor cells that were arranged in cords and plates of various thicknesses that were separated by sinusoid-like areas lined by flat CD34positive endothelial cells. Tumor cells were large with prominent nucleoli and abundant eosinophilic cytoplasm and showed a strong positive staining with Hep Par-1. Immunohistochemistry staining for synaptophysin, chromogranin, CD56, CK7 and CK19 was negative (Fig. 3). These findings corresponded to a moderately differentiated HCC.

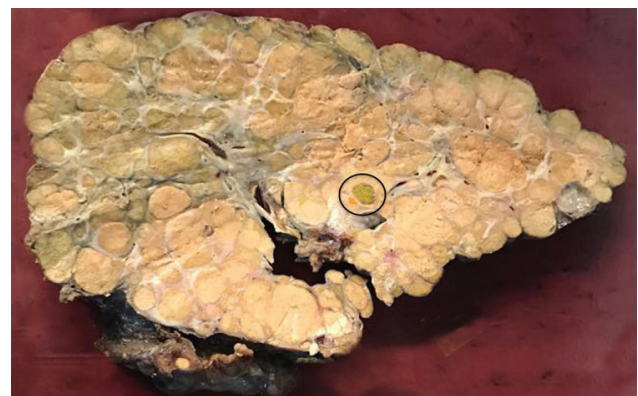

Fig. 2. Cut surface of the liver. A distinct yellowish-green unencapsulated nodular lesion and a different yellow-tan area are barely discriminated (inside the circle) among cirrhotic nodules with different size.

The second smaller distinct component of the tumor was formed from small uniform tumor cells with a narrow cytoplasm and condensed chromatin, giving the nuclei a hyperchromatic appearance. These cells were arranged in a trabecular fashion and as nests. The presence of tumor cells in the vicinity of the ductal structures was also observed. Atypical mitosis was $5 / 10$ in high power field. Immunohistochemistry staining showed positivity for chromogranin, synaptophysin, NSE, CD56 and CK7, but negativity for Hep Par1 and CK19. For MIB-1, immunostaining of the endocrine component indicated a proliferative activity of $18 \%$. These findings were in accordance with NEC grade 2 (G2) (Fig. 3). A broad fibrous band without a transition area separated the tumors from each other. While a strong $\beta$-catenin membrane staining was observed in the HCC area, the NEC area did not show such staining (Fig. 3).

\section{Discussion}

The case presented here was diagnosed as collision tumor type of HCC-NEC, according to the pathological findings. Although two tumors were observed concurrently, a transition between them was not observed. ${ }^{6-10}$ These findings are in contrast with combined tumors that consist of two distinct tumors that are contiguous and intermingle with each other. ${ }^{11-19,21}$ Another important problem to be solved in making a diagnosis of primary hepatic NEC is the discrimination of primary NEC and from metastatic NEC, as the liver is the most frequent site of metastasis for NEC. ${ }^{22}$ Therefore other primary sites should be examined when an NEC is suspected in the liver. ${ }^{14}$ In our case, there were no other neoplastic lesions found besides in the liver.

The clinical and pathologic findings of the cases described in the literature are presented in Tables 1 and 2 . The present case involved a 56-year-old man who had undergone liver transplantation because of chronic liver failure. The diagnosis of a collision type of HCC-NEC was made after the pathological examination of the resection specimen. The age and sex of the patient are in agreement with previously reported cases (mean: 64.4 years; male/female: 20/1) and support that these tumors are more frequently seen in older age groups and men.

Because many patients are followed up for chronic viral hepatitis (hepatitis B virus, 30\%; hepatitis C virus, 60\%), tumor-related symptoms are absent in most cases (50\%). In symptomatic cases, abdominal pain is the most common finding (Table 1). Our patient was being followed up for cirrhosis related to alcoholic hepatitis and did not have any tumorrelated symptoms. Laboratory investigation of tumor markers in recently reported cases demonstrated that AFP was frequently high in $76 \%$ of the cases. On the other hand, similar 
Yılmaz D.B. et al: Incidental collision tumor of HCC-NEC
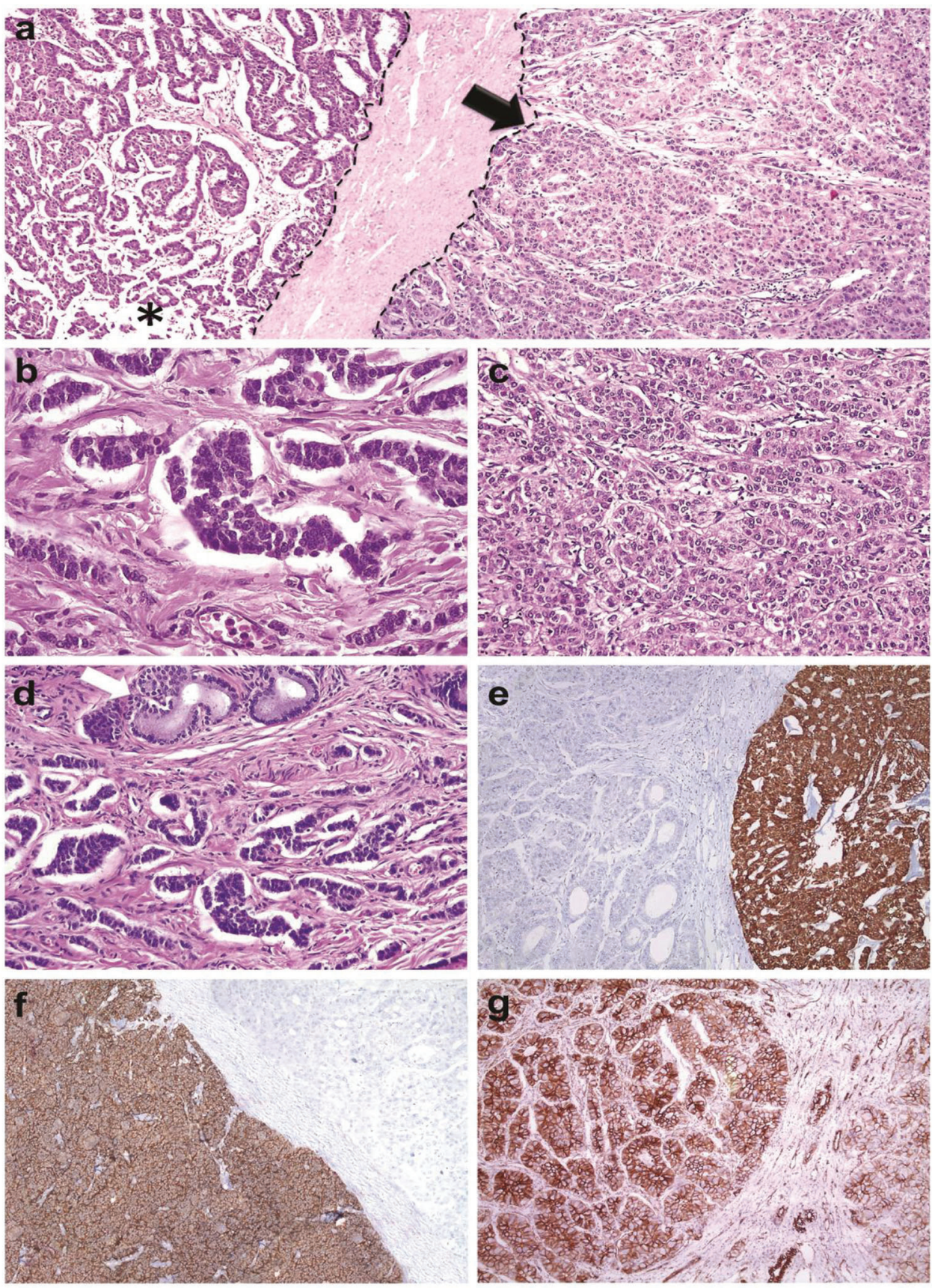

Fig. 3. a: The two different components are separated by a fibrous tissue (tumor margins are highlighted by dotted lines). Hepatocellular carcinoma is indicated by the arrow; asterisk indicates NEC areas. b: Higher magnification of endocrine tumor. c: Higher magnification of HCC. d: NEC cells are closely located to near the ductus. e: NEC stains with chromogranin, but HCC remains negative. f: Hep-par 1 expression detected in HCC, but NEC is negative. g: Strong membrane staining with $\beta$-catenin in HCC.

to the case presented here, AFP was within normal ranges in $19 \%$ of cases. Other tumor markers, such as carcinoembryonic antigen, $7,14-16,18,19$ carbohydrate antigen-19-97,8,15,16,18,19 and carbohydrate antigen-1257,18 were not found to be predictive in the diagnosis of these tumors. PIVKA-II (protein induced by vitamin $\mathrm{K}$ deficiency or antagonists-II) level was normal in a case reported by Nakanishi et al. ${ }^{16}$ Only one case displayed a high level of PIVKA-II. ${ }^{19}$

These data indicate that although these markers are valuable in the discrimination of malignant tumors, they cannot completely rule out some malignancies, such as combined or collision type of HCC-NEC, especially in which cases the AFP level might be normal. Radiological findings of these lesions are not specific and their discrimination is not always possible. Parallel to these observations, in our case neither the tumor nor its nature could be clarified by the radiological investigation. In $80 \%$ of the recently reported cases, the diagnosis necessitated surgical resection. Only 2 cases were diagnosed by fine needle aspiration and liver biopsy and 1 case was diagnosed at autopsy (Table 1). Therefore, the diagnosis of these tumors relies on 
Yılmaz D.B. et al: Incidental collision tumor of HCC-NEC

Table 1. Demographic, laboratory, clinical and macroscopic findings of previously reported HCC-NEC cases

\begin{tabular}{|c|c|c|c|c|c|c|c|c|}
\hline Reference, year & Age & Sex & Symptoms & Virus & AFP & Diagnosis & Metastasis & $\mathrm{D}$, in $\mathrm{cm}$ \\
\hline Barsky et al. ${ }^{11}, 1984$ & 43 & $M$ & Abdominal swelling & HBV & $\uparrow$ & Autopsy & Omentum & $\mathrm{L}$ \\
\hline Artopoulos et al. ${ }^{12}, 1994$ & 69 & M & Abdominal pain & HBV & $\uparrow$ & FNA & - & 10 \\
\hline Vora et al. ${ }^{13}, 2000$ & 63 & M & $\begin{array}{l}\text { Abdominal pain, } \\
\text { jaundice }\end{array}$ & NA & NA & NA & NA & 10 \\
\hline Ishida et al. ${ }^{6}, 2003$ & 72 & M & - & $\mathrm{HCV}$ & $\uparrow$ & Resection, LND & LN & 3 \\
\hline Yamaguchi et al. ${ }^{14}, 2004$ & 71 & M & - & $\mathrm{HCV}$ & $\uparrow$ & Resection & - & 4.1 \\
\hline Garcia et al. ${ }^{7}, 2006$ & 50 & M & - & $\mathrm{HCV}$ & $\uparrow$ & Resection & - & 5.3 \\
\hline Yang et al. ${ }^{15}, 2009$ & 65 & M & Epigastric pain & HBV & $\rightarrow$ & Resection, LND & LN & 7.5 \\
\hline Tazi et al. ${ }^{8}, 2011$ & 68 & M & - & HBV & $\uparrow$ & Resection & - & 4.0 \\
\hline Nakanishi et al. ${ }^{16}, 2012$ & 76 & M & - & $\mathrm{HCV}$ & $\uparrow$ & Resection & - & 3.0 \\
\hline Hammedi et al. ${ }^{17}, 2012$ & 51 & M & Abdominal pain & $\mathrm{HCV}$ & $\uparrow$ & Resection & - & 20 \\
\hline Aboelenen et al. ${ }^{18}, 2014$ & 56 & $\mathrm{~F}$ & $\begin{array}{l}\text { Abdominal } \\
\text { distension }\end{array}$ & - & $\uparrow$ & Biopsy & LN & NA \\
\hline Nishino et al. ${ }^{19}, 2016$ & 72 & M & - & $\mathrm{HCV}$ & $\uparrow$ & Resection & - & 2.5 \\
\hline Choi et al. ${ }^{9}, 2016$ & 72 & M & - & $\mathrm{HCV}$ & $\rightarrow$ & Resection & - & 2.5 \\
\hline \multirow[t]{5}{*}{ Nomura et al. ${ }^{5}, 2016$} & 71 & M & NA & $\mathrm{HCV}$ & $\uparrow$ & Resection & NA & 4 \\
\hline & 71 & M & NA & $\mathrm{HCV}$ & $\rightarrow$ & Resection & NA & 3 \\
\hline & 58 & M & NA & HBV & $\uparrow$ & Resection & NA & 4.3 \\
\hline & 50 & M & NA & HBV & $\uparrow$ & Resection & NA & 1.8 \\
\hline & 63 & M & NA & $\mathrm{HCV}$ & $\uparrow$ & Resection & NA & 3 \\
\hline Baker et al. ${ }^{21}, 2016$ & 76 & M & NA & - & $\uparrow$ & Resection & - & 5.5 \\
\hline Liu et al. ${ }^{10}, 2016$ & 65 & M & $\begin{array}{l}\text { Abdominal } \\
\text { discomfort }\end{array}$ & $\mathrm{HCV}$ & $\uparrow$ & Resection & LN & 4.3 \\
\hline Okumura et al. ${ }^{20}, 2017$ & 70 & M & Solid mass & $\mathrm{HCV}$ & $\rightarrow$ & Resection & - & 11 \\
\hline Present case & 56 & M & Incidental & - & $\rightarrow$ & Resection & - & 2.3 \\
\hline
\end{tabular}

Abbreviations: -, none; AFP, alpha-fetoprotein; D, diameter; F, female; M, male; NA, not available; L, very large; LND, lymph node dissection.

a comprehensive macroscopic and microscopic evaluation together with additional methods, including immunohistochemistry.

Although multiple HCC-NEC of the liver has been described in some reports, ${ }^{6,9,14}$ they are solitary lesions and frequently do not metastasize at the time of diagnosis, as seen in our case. Rarely, metastases to lymph nodes and omentum have been described (Table 2) ${ }^{6,10,11,15,18}$ An important problem in these cases is the difficulty to detect which component of the tumor determines the behavior of the tumor. In a great majority of recent cases, only the NEC component has been detected in the metastatic focus. ${ }^{7,8,14-16,19}$ This observation suggested that NEC components strongly affect the aggressive behavior of the tumor and it is proposed that the NEC component may be responsible for the poor prognosis in these patients. ${ }^{16,19}$ Recently, Choi et al. ${ }^{9}$ proposed adjuvant chemotherapy to increase life expectancy when the grade of NEC is high. Because the awareness of an NEC component might influence the treatment strategy and eventually the outcome of the patient, ${ }^{9,16,19}$ the possibility of NEC should be considered in HCCs with nodal metastasis.

According to the literature, the mean diameter of HCC-NEC is $6.23 \mathrm{~cm}$ (Table 1). The mean diameter of the tumor presented here was $2.3 \mathrm{~cm}$. When combined type and collision type HCC-NEC are analyzed separately, the mean diameter of combined type tumors $(6.2 \mathrm{~cm})$ is larger than that of collision tumors $(3.94 \mathrm{~cm})$. However, with the few cases reported it is not possible to make a definitive conclusion about this finding. On the other hand, this data indicates that a careful macroscopic examination has paramount importance in the detection of HCC-NEC in resection specimens from patients who suffer from chronic liver diseases.

A significant number of reported HCC-NEC cases (62\%) have developed in noncirrhotic livers (Table 2). In our case, the presence of the tumor in a cirrhotic background is in contrast to this finding. However, 3 of the 7 reported collision tumors of HCC-NEC were also detected in cirrhotic background. $6,10,20$ These data support that HCC-NEC should be considered either clinically and pathologically in all patients with chronic liver diseases, regardless of the presence of cirrhosis.

In the present case, no recurrence was observed during the follow-up (10 months) in accordance with the follow-up information for the previously reported cases of HCC-NEC (Table 2). However, 7 of 12 cases of combined tumor of HCC-NEC with follow-up information reportedly died from the disease (Table 2). The mean survival time is 11.9 months for all reported cases (Fig. 4). Although these data point to an aggressive behavior of these tumors, more cases should be reported to delineate the behavioral difference between combined type and collision type of HCC-NEC. Unfortunately, there is no 
Yılmaz D.B. et al: Incidental collision tumor of HCC-NEC

Table 2. Pathological and clinical findings of previously reported HCC-NEC cases

\begin{tabular}{|c|c|c|c|c|c|c|c|}
\hline Reference, year & $\mathrm{N}$ & $\mathrm{C}$ & Type & Therapy & Recurrence & Time* & ST \\
\hline Barsky et al. ${ }^{11}, 1984$ & $\mathrm{~S}$ & + & Combined & Adriamycin, 5-FU & Unresectable & 26 & $\mathrm{D}$ \\
\hline Artopoulos et al. ${ }^{12}, 1994$ & $\mathrm{~S}$ & + & Combined & - & NA & NA & NA \\
\hline Vora et al. ${ }^{13}, 2000$ & $\mathrm{~S}$ & + & Combined & - & NA & 1 & $\mathrm{D}$ \\
\hline Ishida et $a .^{6}, 2003$ & M & + & Collision & - & NA & NA & NA \\
\hline Yamaguchi et al. ${ }^{14}, 2004$ & M & - & Combined & - & Pelvic bone & 5 & $A$ \\
\hline Garcia et al. ${ }^{7}, 2006$ & $\mathrm{~S}$ & - & Collision & TACE, doxorubicin & Liver, peritoneum & 16 & $A$ \\
\hline Yang et al. ${ }^{15}, 2009$ & $\mathrm{~S}$ & - & Combined & - & $\begin{array}{l}\text { Liver, adrenals, } \\
\text { paraaortic LNs }\end{array}$ & 12 & $\mathrm{D}$ \\
\hline Tazi et al. ${ }^{8}, 2011$ & $\mathrm{~S}$ & - & Collision & Cisplatin, etoposide & - & 28 & $A$ \\
\hline Nakanishi et al. ${ }^{16}, 2012$ & $\mathrm{~S}$ & NA & Combined & $\begin{array}{l}\text { TACE, epirubicin } \\
\text { lipiodol }\end{array}$ & Sacral bone & 17 & $\mathrm{D}$ \\
\hline Hammedi et al. ${ }^{17}, 2012$ & $\mathrm{~S}$ & - & Combined & - & - & 6 & $A$ \\
\hline Aboelenen et al. ${ }^{18}, 2014$ & $\mathrm{~S}$ & - & Combined & - & - & 1 & $\mathrm{D}$ \\
\hline Nishino et al. ${ }^{19}, 2016$ & & - & Combined & Cisplatin, etoposide & $\begin{array}{l}\text { Regional, } \\
\text { paraaortic LNs }\end{array}$ & 2 & $\mathrm{D}$ \\
\hline Choi et al. ${ }^{9}, 2016$ & M & - & Collision & Cisplatin, etoposide & Liver & 10 & $A$ \\
\hline \multirow[t]{5}{*}{ Nomura et al. ${ }^{5}, 2016$} & $\mathrm{~S}$ & - & Combined & - & Liver & 8.6 & $D$ \\
\hline & $\mathrm{S}$ & - & Collision & - & Diaphragm, liver & 2.6 & $D$ \\
\hline & $\mathrm{S}$ & - & Combined & - & - & 19.7 & $A$ \\
\hline & $\mathrm{S}$ & + & Combined & - & - & 19.5 & $A$ \\
\hline & $\mathrm{S}$ & - & Combined & & - & 24 & $A$ \\
\hline Baker et al. ${ }^{21}, 2016$ & $\mathrm{~S}$ & + & Collision & Cisplatin & - & NA & $A$ \\
\hline Liu et al. ${ }^{10}, 2016$ & $\mathrm{~S}$ & + & Collision & - & NA & 1.3 & $\mathrm{D}$ \\
\hline Okumura et al. ${ }^{20}, 2017$ & $\mathrm{~S}$ & - & $\begin{array}{l}\text { Collision } \\
\text { Combined }\end{array}$ & - & LNs & 3 & $\mathrm{D}$ \\
\hline Present case & $\mathrm{S}$ & + & Collision & - & - & 10 & $A$ \\
\hline
\end{tabular}

Abbreviations: *, time in months; +, present; -, none; 5-FU, 5- fluorourasil; A, alive; C, cirrhosis; D, death; LNs, lymph nodes; M, multiple; N, number; NA, not available; S, solitary; ST, status; TACE, transarterial chemoembolization.

specific treatment protocol due to the small number of cases (Table 2).

Although the origin of primary NEC in the liver is not clarified, two hypotheses have been proposed recently. The first hypothesis is that these tumors originate from hepatic

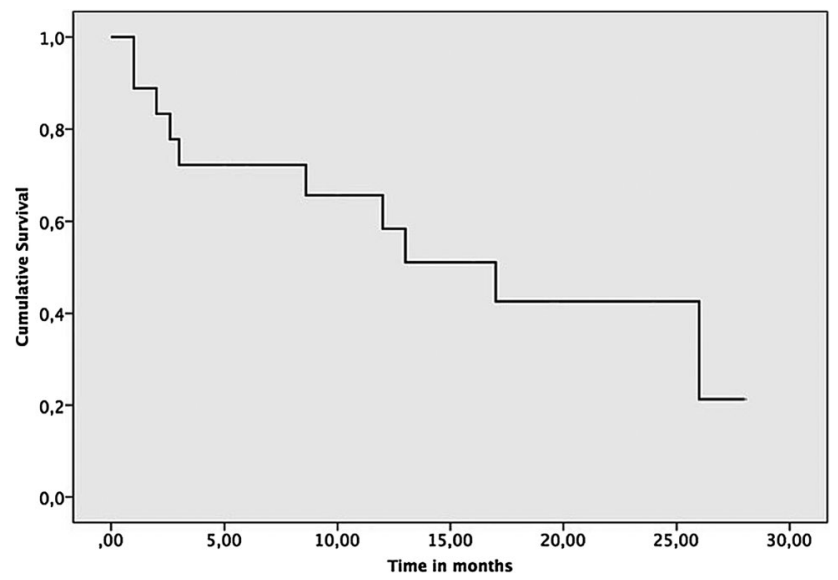

Fig. 4. Cumulative survival of previously reported cases of HCC-NEC. stem cells from intrahepatic bile ducts. ${ }^{23,24}$ This hypothesis is supported by the occurrence of neuroendocrine tumors in noncirrhotic livers. In a recent report, CK19 expression was observed in the NEC portion of the tumor, supporting the role of hepatic stem cells in the evolution of primary NEC. ${ }^{7}$ Although in our case CK19 was negative, the localization of tumor cells in the vicinity of ductal structures was an interesting finding. The other hypothesis is neuroendocrine differentiation of stem cell precursors of malignant cells from another liver tumor. Parallel to this opinion, many HCC with neuroendocrine features have been reported. ${ }^{25}$ Moreover, the expression of neuroendocrine markers in the HCC component of combined HCC-NEC has been observed. ${ }^{6,15}$ In 1 case, the presence of neurosecretory granules was also detected in the HCC component under electron microscopy. ${ }^{15}$ Therefore, it is possible to postulate that these combined tumors arise from stem cells that evolve into divergent differentiation. On the other hand, such findings did not describe collision type HCC-NEC tumors. Because our case was incidentally observed and the diagnosis relied on histopathological and immunohistochemical findings we did not have the possibility to perform an electron microscopic or molecular investigation.

Recently, in an elegant study, Baker et al. ${ }^{21}$ detected a CTNNB1 gene mutation (S33F located in exon 3) in both 
components of a collision tumor of HCC-NEC and suggested a common cell origin. On the other hand, in a recent report, Liu et al. ${ }^{10}$ did not find similar results by immunohistochemistry. They observed $\beta$-catenin staining in both components of the tumor and stated that these findings did not support exon 3 mutations in the CTNNB1 gene. In our case, similar to the case presented by Liu et al., ${ }^{10}$ a strong $\beta$-catenin staining was observed in the HCC component. However, we did not observe $\beta$-catenin expression in the NEC component, leading us to speculate that in these tumors, divergent underlying molecular pathways might take place that involve the $\beta$-catenin pathway. Moreover, a previous case reported by Okumura et al. ${ }^{21}$ did not only show that the composite and collision type HCC-NEC could be synchronous but also indicated that understanding of the histogenesis of these tumors is very difficult to define.

In conclusion, although collision tumor of HCC-NEC of the liver is very rare, the case presented here supports their presence in this localization in older age groups and in men. Moreover, the present case and the review show that the rare occurrence of HCC-NEC of the liver and lack of diagnostic clinical signs and symptoms do not exclude their consideration in the differential diagnosis of liver tumors, especially in patients with the chronic liver disease.

\section{Conflict of interest}

The authors have no conflict of interests related to this publication.

\section{Author contributions}

Study design, analysis and interpretation of data, technical or material support, manuscript writing, and critical revision (DBY, ZB, GÜ, EA, CIBB, GÖE), administration (DBY, ZB, GÜ, EA).

\section{References}

[1] Theise ND, Curado MP, Franceschi S. Bosman FT, Carneiro F, Hruban RH, et al. Hepatocellular carcinoma. WHO Classification of Tumours of the Digestive System 4th ed, Lyon: IARC Press, 2010;205-216.

[2] Goodman ZD, Ishak KG, Langloss JM, Sesterhenn IA, Rabin L. Combined hepatocellular-cholangiocarcinoma. A histologic and immunohistochemica study. Cancer 1985;55:124-135. doi: 10.1002/1097-0142(19850101)55: $1<124:$ :AID-CNCR2820550120>3.0.CO;2-Z

[3] Jarnagin WR, Weber S, Tickoo SK, Koea JB, Obiekwe S, Fong Y, et al. Combined hepatocellular and cholangiocarcinoma: demographic, clinical, and prognostic factors. Cancer 2002;94:2040-2046. doi: 10.1002/cncr.10392

[4] Haratake J, Hashimoto H. An immunohistochemical analysis of 13 cases with combined hepatocellular and cholangiocellular carcinoma. Liver 1995;15: 9-15. doi: 10.1111/j.1600-0676.1995.tb00099.x

[5] Nomura Y, Nakashima O, Akiba J, Ogasawara S, Fukutomi S, Yamaguchi R, et al. Clinicopathological features of neoplasms with neuroendocrine differentiation occurring in the liver. J Clin Pathol 2017;70:563-570. doi: 10.1136/jclinpath2016-203941.

[6] Ishida M, Seki K, Tatsuzawa A, Katayama K, Hirose K, Azuma T, et al. Primary hepatic neuroendocrine carcinoma coexisting with hepatocellular carcinoma in hepatitis C liver cirrhosis: report of a case. Surg Today 2003;33:214-218. doi: $10.1007 / \mathrm{s} 005950300048$

[7] Garcia MT, Bejarano PA, Yssa M, Buitrago E, Livingstone A. Tumor of the liver (hepatocellular and high grade neuroendocrine carcinoma): a case report and review of the literature. Virchows Arch 2006;449:376-381. doi: 10 1007/s00428-006-0251-0.

[8] Tazi EM, Essadi I, M'rabti H, Errihani H. Hepatocellular carcinoma and high grade neuroendocrine carcinoma: a case report and review of the literature. World J Oncol 2011;2:37-40. doi: 10.4021/wjon276e.

[9] Choi GH, Ann SY, Lee SI, Kim SB, Song IH. Collision tumor of hepatocellular carcinoma and neuroendocrine carcinoma involving the liver: Case report and review of the literature. World J Gastroenterol 2016;22:9229-9234. doi: $10.3748 / w j g . v 22 . i 41.9229$.

[10] Liu Y], Ng KF, Huang SC, Wu RC, Chen TC. Composite hepatocellular carcinoma and small cell carcinoma with early nodal metastasis: A case report. Medicine (Baltimore) 2017;96:e7868. doi: 10.1097/MD.0000000000007868.

[11] Barsky SH, Linnoila I, Triche TJ, Costa J. Hepatocellular carcinoma with carcinoid features. Hum Pathol 1984;15:892-894. doi: 10.1016/S0046-8177 (84)80152-5

[12] Artopoulos JG, Destuni C. Primary mixed hepatocellular carcinoma with carcinoid characteristics. A case report. Hepatogastroenterology 1994;41:442444

[13] Vora IM, Amarapurkar AD, Rege JD, Mathur SK. Neuroendocrine differentiation in hepatocellular carcinoma. Indian J Gastroenterol 2000;19:37-38.

[14] Yamaguchi R, Nakashima O, Ogata T, Hanada K, Kumabe T, Kojiro M. Hepatocellular carcinoma with an unusual neuroendocrine component. Pathol Int 2004;54:861-865. doi: 10.1111/j.1440-1827.2004.01770.x.

[15] Yang CS, Wen MC, Jan YJ, Wang J, Wu CC. Combined primary neuroendocrine carcinoma and hepatocellular carcinoma of the liver. J Chin Med Assoc 2009;72:430-433. doi: 10.1016/S1726-4901(09)70400-9.

[16] Nakanishi C, Sato K, Ito Y, Abe T, Akada T, Muto R, et al. Combined hepatocellular carcinoma and neuroendocrine carcinoma with sarcomatous change of the liver after transarterial chemoembolization. Hepatol Res 2012;42: 1141-1145. doi: 10.1111/j.1872-034X.2012.01017.x.

[17] Hammedi F, Rammah S, Trabelsi A, Bdioui A, Jomaa W, Anjorin A, et al. Carcinome hépatocellulaire avec composante neuroendocrine: à propos d'un cas. J Afr Cancer 2012;4:120-123. doi: 10.1007/s12558-011-0175-8.

[18] Aboelenen A, El-Hawary AK, Megahed N, Zalata KR, El-Salk EM, Fattah MA, et al. Right hepatectomy for combined primary neuroendocrine and hepatocellular carcinoma. A case report. Int J Surg Case Rep 2014;5:26-29. doi: 10.1016/j.ijscr.2013.10.018.

[19] Nishino H, Hatano E, Seo S, Shibuya S, Anazawa T, Iida T, et al. Histological features of mixed neuroendocrine carcinoma and hepatocellular carcinoma in the liver: a case report and literature review. Clin J Gastroenterol 2016;9: 272-279. doi: 10.1007/s12328-016-0669-0

[20] Okumura $Y$, Kohashi K, Wang $H$, Kato M, Maehara $Y$, Ogawa $Y$, et al. Combined primary hepatic neuroendocrine carcinoma and hepatocellular carcinoma with aggressive biological behavior (adverse clinical course): A case report. Patho Res Pract 2017;213:1322-1326. doi: 10.1016/j.prp.2017.06.001.

[21] Baker E, Jacobs C, Martinie J, Iannitti DA, Vrochides D, Swan RZ. Mixed hepatocellular carcinoma, neuroendocrine carcinoma of the liver. Am Surg 2016;82:1121-1125.

[22] Gurung A, Yoshida EM, Scudamore CH, Hashim A, Erb SR, Webber DL. Primary hepatic neuroendocrine tumour requiring live donor liver transplantation: case report and concise review. Ann Hepatol 2012;11:715-720.

[23] Roskams T, van den Oord J], De Vos R, Desmet V]. Neuroendocrine features of reactive bile ductules in cholestatic liver disease. Am J Pathol 1990;137 1019-1025.

[24] Roskams T, Cassiman D, De Vos R, Libbrecht L. Neuroregulation of the neuroendocrine compartment of the liver. Anat Rec A Discov Mol Cell Evol Biol 2004;280:910-923. doi: 10.1002/ar.a.20096

[25] Zhao M, Laissue JA, Zimmermann A. "Neuroendocrine" differentiation in hepatocellular carcinomas (HCCs): immunohistochemical reactivity is related to distinct tumor cell types, but not to tumor grade. Histol Histopathol $1993 ; 8: 617-626$. 